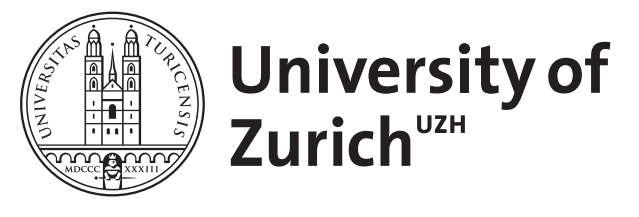

\title{
Historische Pragmatik
}

\author{
Jucker, Andreas H
}

\begin{abstract}
Historische Pragmatik`bezeichnet ein eigenständiges Teilgebiet der Pragmatik, das sich seit den 1990er Jahren stark entwickelt und ausdifferenziert hat. Es befasst sich im Wesentlichen mit pragmatischen Fragestellungen an historischem Sprachmaterial. Fragen der Sprachverwendung werden zusammengebracht mit Fragen der historischen Linguistik zu früheren Sprachformen, zur Entwicklung von Sprache und Sprachen und somit ganz generell zu den Fragen des Sprachwandels. Die historische Pragmatik setzt in den meisten Fällen ein Verständnis von Pragmatik voraus, bei dem es ganz wesentlich auch um den sozialen Kontext der Sprachverwendung geht. Dieses Verständnis von Pragmatik wird oft unter dem Begriff 〉Soziopragmatik`subsumiert und orientiert sich nicht unwesentlich an Arbeiten in der Soziologie und Anthropologie.
\end{abstract}

DOI: https://doi.org/10.1007/978-3-476-04624-6_13

Posted at the Zurich Open Repository and Archive, University of Zurich ZORA URL: https://doi.org/10.5167/uzh-169339

Book Section

Accepted Version

Originally published at:

Jucker, Andreas H (2018). Historische Pragmatik. In: Liedtke, Frank; Tuchen, Astrid. Handbuch Pragmatik. Stuttgart: J. B. Metzler, 132-139.

DOI: https://doi.org/10.1007/978-3-476-04624-6_13 
Eingereicht Version. Erschienen in: Liedtke, Frank, und Astrid Tuchen (Hg.) (2018)

Handbuch Pragmatik. Stuttgart: J. B. Metzler, 132-139.

\section{Historische Pragmatik}

\section{Begriffsbestimmung}

Historische Pragmatik bezeichnet ein eigenständiges Teilgebiet der Pragmatik, das sich seit den Neunzigerjahren des letzten Jahrhunderts stark entwickelt und ausdifferenziert hat. Es befasst sich im Wesentlichen mit pragmatischen Fragestellungen an historischem Sprachmaterial. Fragen der Sprachverwendung werden zusammengebracht mit Fragen der historischen Linguistik zu früheren Sprachformen, zur Entwicklung von Sprache und Sprachen und somit ganz generell zu den Fragen des Sprachwandels. Die historische Pragmatik setzt in den meisten Fällen ein Verständnis von Pragmatik voraus, bei dem es ganz wesentlich auch um den sozialen Kontext der Sprachverwendung geht. Dieses Verständnis von Pragmatik wird oft unter dem Begriff Soziopragmatik subsumiert und orientiert sich nicht unwesentlich an Arbeiten in der Soziologie und Anthropologie. Die theoretische Pragmatik dagegen ist enger auf die eigentlichen Kommunikationsprozesse ausgerichtet, aber ohne deren soziale Einbettung und beruft sich oft auf Arbeiten aus dem Bereich der Sprachphilosophie (siehe dazu insbesondere Chapman 2011: 15).

Verschiedene Zugänge können in der historischen Pragmatik unterschieden werden. In einem ersten Zugang, den man als pragmatische Sprachgeschichte bezeichnen kann, wird die Entwicklung einer Sprache als Resultat von kommunikativen Handlungen in einem sozialen Kontext betrachtet. Sprachwandel und Sprachgeschichte werden hier sozusagen mit einem pragmatischen Instrumentarium untersucht. Dieser Zugang geht ganz wesentlich davon aus, dass es bei dem, was Systemlinguistinnen und -linguisten als Sprachwandel bezeichnen, im eigentlichen Sinne um einen Wandel der Sprachverwendung geht. Sprecherinnen und Sprecher verwenden in ihrem alltäglichen Sprachgebrauch immer mal wieder Wörter mit einer neuen oder angepassten Bedeutung, mit einer eigenwilligen Aussprache oder in einem ungewöhnlichen syntaktischen Kontext. Gelegentlich werden solche und ähnliche Idiosynkrasien von anderen Sprecherinnen und Sprechern übernommen und weiterverbreitet. Bei einer ausreichenden Verbreitung werden solche Idiosynkrasien zu Konventionen. Die Sprachverwendung hat sich punktuell geändert und aus der Außensicht wird ein Sprachwandel wahrnehmbar. Solche Änderungen der Sprachverwendung werden von 
der pragmatischen Sprachgeschichte als Konsequenzen von kommunikativen Handlungen untersucht und beschrieben. Die pragmatische Sprachgeschichtsschreibung verwendet konsequenterweise ein Instrumentarium von pragmalinguistischen Konzepten, um diese Phänomene zu erklären.

Beim zweiten Zugang zur historischen Pragmatik, den man als historische Sprachpragmatik bezeichnen kann, steht die Sprachverwendung selber im Zentrum des wissenschaftlichen Interessens. Die Fragestellungen der Pragmatik, die üblicherweise an der Gegenwartssprache untersucht werden, werden hier auf historische Sprachstufen übertragen, seien das Fragen der Verwendung von Sprechakten oder Diskurspartikeln, Fragen der Höflichkeit oder Unhöflichkeit, Fragen des inferenzbasierten Sprachverstehens und so weiter. Eine solche Übertragung von Fragestellungen von der Gegenwartssprache auf historische Sprachstufen ist nicht unproblematisch, da die Datenlage und damit die zur Verfügung stehenden Analysemethoden deutlich verschieden sind. Darauf wird in Abschnitt 3 unten näher einzugehen sein.

In der historischen Sprachpragmatik kann man weiterhin unterscheiden zwischen synchronen, kontrastiven und diachronen Ansätzen. In einer synchronen historischen Sprachpragmatik geht es um die Beschreibung der Sprachverwendung zu einem beliebigen historischen Zeitpunkt. Das mögen zum Beispiel Untersuchungen zur Sprachverwendung in einem Theaterstück der englischen Renaissance, in einer Sammlung von Briefen aus dem Alten Ägypten oder in deutschen Gerichtsprotokollen aus dem siebzehnten Jahrhundert sein, in denen es in erster Linie um die konkrete Sprachverwendung zu diesem historischen Zeitpunkt in den untersuchten Quellen geht ohne einen Vergleich zu früheren oder späteren Epochen.

In der kontrastiven historischen Sprachpragmatik dagegen rückt der Vergleich zwischen zwei oder mehreren Epochen in das Zentrum der Untersuchung. Hier besteht ein enger Zusammenhang zwischen der historischen Pragmatik und der kontrastiven Pragmatik, die die Sprachverwendung in zwei verschiedenen (Gegenwarts-)Sprachen oder Sprachvarietäten untersucht. In beiden Fällen stellen sich Fragen der Vergleichbarkeit. Ein Vergleich, sei es über die Sprachgrenzen unterschiedlicher Sprachen oder über die Sprachstufen einer einzelnen Sprache setzen ein Tertium Comparationis, also eine Gemeinsamkeit der zu vergleichenden Entitäten voraus. Ein Vergleich einer Entschuldigung oder eines Kompliments im Japanischen und im Deutschen setzt voraus, dass sich diese Sprechakte 
unabhängig von ihrer jeweiligen Realisierung in den beiden Sprachen in funktioneller Hinsicht auch tatsächlich entsprechen. Dasselbe gilt für einen Vergleich dieser Sprechakte in der frühen Neuzeit mit ihren Entsprechungen in der Gegenwartssprache. Ein wesentlicher Unterschied zwischen dem kontrastiven historischen Vergleich und dem kontrastiven Vergleich über zeitgenössische Sprachgrenzen hinweg besteht darin, dass im ersten Fall häufig eine Entwicklungslinie vom früheren Sprachstand zum späteren postuliert werden kann, während beim Sprachvergleich allenfalls ein gemeinsamer historischer Ursprung vorliegt, aber in der Regel keine offensichtliche Entwicklungslinie von der einen Situation zur anderen.

In der diachronen historischen Sprachpragmatik schließlich geht es genau um diese Entwicklungslinien zwischen verschiedenen Sprachstufen. Hier geht es nicht mehr um einen Vergleich zwischen früher und später, sondern um die Details, wie sich das Früher in das Später gewandelt hat, welche kommunikativen Kräfte den Wandel ermöglicht oder begünstigt haben und wie sich der Wandel in der entsprechenden Sprachgemeinschaft ausgebreitet hat. An diesem Punkt kommen in der Regel pragmalinguistische Erklärungsansätze zum Zuge, wie sie oben im Zusammenhang mit der pragmatischen Sprachgeschichte erwähnt wurden, womit sich die pragmatische Sprachgeschichte mit der historischen Sprachpragmatik vereint.

Im Folgenden werde ich kurz auf die Anfänge der historischen Pragmatik eingehen, bevor ich mich dann in Abschnitt 3 den Fragen der für die historische Pragmatik zur Verfügung stehenden Daten und den für die Untersuchungen geeigneten Methoden zuwende. Abschnitt 4 stellt einige neuere Arbeiten aus ganz unterschiedlichen philologischen $\mathrm{Zu}-$ sammenhängen vor. Sie sollen exemplarisch die Breite und die Vielfältigkeit der gegenwärtigen Forschung in der historischen Pragmatik aufzeigen. Der letzte Abschnitt gibt einen kurzen Ausblick auf die weitere Entwicklung der historischen Pragmatik.

\section{Die Anfänge der historischen Pragmatik}

Untersuchungen, die man aus heutiger Sicht der historischen Pragmatik zurechnen würde, reichen sehr weit zurück, auch wenn es sich dabei jeweils um vereinzelte Arbeiten handelte, die nicht im Kontext eines entsprechenden Forschungsparadigmas entstanden. So befasst sich zum Beispiel bereits Stroebe (1911) mit altgermanischen Grußformen und somit mit einer Ausprägung eines ganz bestimmten Sprechaktes, dem Sprechakt des Grüßens, 
in einem historischen Kontext. Den Grußformeln im Altfranzösischen widmet sich Lebsanft (1988). Neben dem Grüßen fand vor allem das Fluchen und Beschimpfen in historischen Kontexten schon früh die Beachtung in der historischen Linguistik, so zum Beispiel das Fluchen und Beschimpfen im Schweizerdeutschen (Lötscher 1981) oder Beleidigungen im Paris des achtzehnten Jahrhunderts (Garrioch 1987). Montagu (1967) legte eine eigentliche Kulturgeschichte des Fluchens vor, die das Fluchen in all seinen Ausprägungen von den Antiken Ägyptern, Griechen und Römer über das Frühneuenglische Shakespeares bis zum gegenwärtigen amerikanischen Englischen untersuchte.

Daneben gab es auch schon relativ früh theoretische Arbeiten zur Historizität von Sprechakten. Schlieben-Lange (1976) vertrat in einem vielbeachteten Aufsatz die These, dass es keine universellen Sprachhandlungen gebe, sondern nur jeweils historisch bedingte und ausgeprägte, konventionalisierte sprachliche Handlungen (1976: 114). Daraus folgte für sie, dass es einen Sprechakt nur dann geben kann, wenn es in einer Sprachgemeinschaft dafür eine konkrete Bezeichnung gibt (siehe auch das Streitgespräch dazu in SchliebenLange und Weydt 1979).

Ebenfalls früh begann das Interesse an den Anredeformen in historischen Kontexten. Meistens befassten sich diese Arbeiten mit literarischen Quellen. Auch diese Arbeiten können aus heutiger Sicht im weiteren Sinne der historischen Pragmatik zugerechnet werden. Bereits Ehrismann (1901-1904) und Metcalf (1938) befassten sich mit den Anredepronomina im Deutschen, die nicht nur wie in vielen europäischen Sprachen zwei Formen (zum Beispiel tu und vous im Französischen) sondern vom achtzehnten bis zum frühen neunzehnten Jahrhundert vier oder sogar fünf Formen umfasste $(d u, i h r, e r / s i e$, Sie und dieselben; siehe Simon 2003: 86).

Brown und Gilman (1960) entwarfen einen Erklärungsansatz für die Verwendung der Anredepronomina in einer ganzen Reihe von europäischen Sprachen, insbesondere Englisch, Französisch, Italienisch, Spanisch und Deutsch. Die Verwendung unterschiedlicher Pronomina für die Anrede Einzelner wird als Ausdruck der sozialen Strukturen, der Ideologien der Macht und der Semantik der Pronomina gesehen. Zu den Anredeformen in einzelnen Sprachen liegen zahlreiche frühe Arbeiten vor, so zum Beispiel von Finkenstaedt (1963) und von Breuer (1983) zum Englischen, von Fritz und Muckenhaupt (1981) zum Deutschen, von Friedrich (1986) zum Russischen, von Lebsanft (1987) zum Französischen und von Betsch (1989) zum Tschechischen. 
Als genuine Subdisziplin der Pragmatik konstituierte sich die historische Pragmatik jedoch erst im Laufe der Neunzigerjahre des letzten Jahrhunderts. Verschiedene Faktoren trugen dazu bei, dass sich das Gebiet in dieser Zeit etablieren und ausbreiten konnte. Nach einer Fokussierung der Linguistik in den Sechziger- und Siebzigerjahren auf die Kerngebiete Syntax, Semantik, Morphologie und Phonologie begann sich das Interesse schon in den Achtzigerjahren vermehrt den Performanzelementen der Sprache und insbesondere der gesprochenen Sprache zuzuwenden. Die Interaktion rückte in den Fokus und erforderte eine Abkehr von der Introspektion als Arbeitsmethode zu empirischen Methoden der Datenanalyse. Die Pragmatik etablierte sich als ein zentrales und wichtiges Teilgebiet der Linguistik (siehe Jucker 2012a: 503; Taavitsainen und Jucker 2015). In den Neunzigerjahren kam das erstarkte Interesse an den historischen Zusammenhängen dazu, das durch die Verfügbarkeit der ersten großen historischen Textkorpora beflügelt wurde. Gleichzeitig öffnete sich der Blick der Pragmatik, der sich anfänglich ausschließlich auf gesprochene Daten gerichtet hatte, auch auf Formen der schriftbasierten Kommunikation. Damit ergaben sich neue Voraussetzungen der Zusammenarbeit der historischen Linguistik mit der Pragmatik.

\section{Daten und Methoden}

Die frühen Arbeiten zur historischen Pragmatik beruhten fast ausschließlich auf den herkömmlichen philologischen Methoden genauer Textkenntnisse und umfangreicher Textlektüre. In den Neunzigerjahren des letzten Jahrhunderts veränderte sich die Situation in mehrfacher Weise. Der Sprachwissenschaft standen nun plötzlich umfangreiche elektronische Textkorpora, korpuslinguistische Programme und die dazu notwendige, erschwingliche Computerinfrastruktur zur Verfügung. Die Ausweitung der pragmatischen Untersuchungsgegenstände auf schriftliche Daten machten es möglich, die nun leichter zugänglichen historischen Daten aus einer pragmatischen Perspektive zu untersuchen. Die frühen Jahre der historischen Pragmatik waren allerdings noch sehr stark durch Diskussionen zur Legitimität der schriftlichen Daten geprägt. Lange galten schriftliche Daten mit einer besonderen Nähe zur gesprochenen Sprache als besonders geeignet für historischpragmatische Analysen. Gerichtsprotokolle zum Beispiel enthalten verschriftlichte Versionen mündlicher Interaktion, auch wenn es sich dabei um stark institutionalisierte Interaktion handelt und die Mitschriften nicht in Hinsicht auf die Bedürfnisse der heutigen 
Gesprächsforschung verfasst wurden (siehe dazu zum Beispiel Kryk-Kastovsky 2000; Culpeper und Kytö 2000).

Private Korrespondenz basiert nicht im gleichen Maße auf gesprochener Sprache, aber sie hat den Vorteil der Interaktion zwischen Absender und Adressat, die sich in ihren Rollen abwechseln. Zudem ist die Sprache in aller Regel weit weniger formell und konventionalisiert als etwa in den Gerichtsprotokollen. Briefwechsel wurden und werden deshalb ebenfalls als besonders geeignet erachtet für die historische Pragmatik (siehe insbesondere Nevalainen und Raumolin-Brunberg 1995; Schwitalla 2000; Held 2005).

Bei Dramentexten handelt es sich um fiktive Interaktionen zwischen Gesprächspartnern, deren Verwendbarkeit für die historische Pragmatik aber gerade wegen ihrer Fiktionalität und wegen ihrer literarischen Ansprüche immer wieder kontrovers diskutiert wurde. Ihre Verwendung wurde mit der Begründung gerechtfertigt, dass sie zwar nicht ideal sei, aber als einzig vorliegende Annäherung an die gesprochene Sprache trotzdem in Frage kommt, zumal wenn es sich um Texte herausragender Autoren wie zum Beispiel Shakespeare handle (Salmon 1967; Brown und Gilman 1989).

Heute ist der Umgang mit schriftlichen Daten in der historischen Pragmatik deutlich weniger apologetisch als noch vor zehn oder zwanzig Jahren. Überlieferte Text, seien das Gerichtsprotokolle, Briefe oder Theaterstücke, werden nicht länger als mehr oder weniger geeignete Annäherungen an gesprochene Interaktion angesehen, sondern sie werden in ihren ursprünglichen kommunikativen Zusammenhängen als das untersucht, was sie tatsächlich sind, ohne voreiligen Schlussfolgerungen auf andere kommunikative Zusammenhänge (siehe besonders Jucker und Taavitsainen 2013: 25-26).

Die Einschränkung auf schriftliche Daten in der historischen Pragmatik hat offenkundige Konsequenzen für die Wahl der Methoden. Experimentelle Methoden der Datengenerierung wie Befragungen, Diskursergänzungsaufgaben oder Rollenspiele stehen selbstredend nicht zur Verfügung. Die historische Pragmatik stützt sich deshalb notwendigerweise auf die Methoden der Textanalyse und -interpretation. Das können qualitative Methoden sein, die der philologischen Tradition folgend auf einer sorgfältigen Lektüre und Analyse einer überschaubaren Textmenge basiert, oder es können quantitative Methoden sein, die auf der computergestützten Auswertung großer Textkorpora beruht (siehe Jucker und Taavitsainen 2013: Kapitel 3). 
Vor allem der korpuslinguistischen Arbeitsweise kommt in der historischen Pragmatik in den letzten Jahren ein immer größerer Stellenwert zu (siehe Taavitsainen, Jucker und Tuominen 2014). In den Anfängen der historischen Pragmatik in den Neunzigerjahren des letzten Jahrhunderts beruhten die Arbeiten noch auf relativ kleinen Korpora, wie zum Beispiel dem Helsinkikorpus, der mit weniger als zwei Millionen Wörter die englische Sprache seit ihren Anfängen im siebten und achten Jahrhundert bis in die frühe Neuzeit abzubilden versuchte. Schnell kamen spezialisierte Korpora dazu, die einer einzelnen Textkategorie gewidmet waren wie zum Beispiel brieflicher Korrespondenz oder medizinischen Texten. Heute liegen eine Vielzahl von großen und sehr großen historischen Korpora vor, die entweder eine spezialisierte Textsorte umfassen (zum Beispiel das 134 Millionen Wörter umfassende Old Bailey Corpus mit englischen Gerichtsprotokollen von 1674 bis 1913) oder aber eine nationale Varietät in ihrer ganzen Breite abzubilden versuchen (zum Beispiel das 400 Millionen Wörter umfassende Corpus of Historical American English, das die zwei Jahrhunderte zwischen 1810 bis 2009 abbildet).

Korpuslinguistische Fragestellungen setzen voraus, dass sich relevante sprachliche Elemente identifizieren und in Suchalgorithmen übertragen lassen. In manchen Fällen ist das relativ einfach, so zum Beispiel, wenn es um Diskurspartikel geht, deren Form sich im diachronen Sprachverlauf kaum verändert haben. Hier ist der Ausgangspunkt eine sprachliche Form, deren sich wandelnde Funktion im Laufe der Zeit untersucht werden soll (formto-function mapping). Schwieriger sind die Fragestellungen, die nach sprachlichen Funktionen suchen, um deren sich ändernde sprachliche Ausprägungen zu untersuchen (function-to-form mapping). Das kann zum Beispiel ein spezifischer Sprechakt sein, ein Kompliment, eine Aufforderung oder ein Befehl. In solchen Fällen müssen sich die Suchalgorithmen auf typische sprachliche Elemente des gesuchten Sprechaktes abstützen und riskieren dabei, ungewöhnliche Ausprägungen desselben zu übersehen. Als Alternative dazu kann sich eine historische Sprechaktstudie auch auf das entsprechende Sprechaktverb stützen und über dessen Vorkommen nicht die eigentlichen Manifestationen des Sprechaktes, sondern metakommunikative Äußerungen dazu untersuchen. Komplimente zum Beispiel sind kaum konventionalisiert und lassen sich mit typischen sprachlichen Elementen kaum in ausreichender Zahl finden. Stattdessen lassen sich aber Stellen lokalisieren, in denen die vorliegendene Texte sich explizit zu Komplimenten äußern (siehe Jucker und Taavitsainen 2014). 


\section{Neuere Arbeiten und Themen}

Die Forschungsliteratur der historischen Pragmatik ist in der Zwischenzeit so stark angewachsen, dass eine adäquate Übersicht im Rahmen des vorliegenden Handbuchbeitrags nicht geleistet werden kann. Ich beschränke mich deshalb im Folgenden darauf, eine kleine Auswahl aktueller Arbeiten vorzustellen, die exemplarisch für verschiedene Forschungsparadigmen der historischen Pragmatik stehen mögen. Es geht mir dabei in erster Linie darum, die Vielfältigkeit der Fragestellungen, der verwendeten Methoden und nicht zuletzt auch der untersuchten Sprachen zu illustrieren.

Traugott (2016) untersucht pragmatische Markierungen an der rechten Satzperipherie, also am Ende eines Satzes oder Teilsatzes. Bisher sind diese Elemente fast ausschließlich an der linken Peripherie, also am Satzanfang oder im Innern des Satzes untersucht worden. Es geht ihr dabei vor allem darum aufzuzeigen, wie sich das Inventar verschiedener Typen im Verlaufe der englischen Sprachgeschichte vom Altenglischen bis zum Gegenwartsenglisch in dieser Satzposition entwickelt hat. Die längste Geschichte haben epistemische Adverbien wie no doubt oder surely, die in der Form witodlice oder soðlice ,bestimmt, wirklich' bereits im Altenglischen an der rechten Satzperipherie nachgewiesen werden können. Im Mittelenglischen kamen generalisierte Listenerweiterungen wie and stuff und or something, sowie Kommentareinschübe wie I think, y'know und see bzw. deren mittelenglischen Entsprechungen dazu. Im weiteren Verlauf der englischen Sprachgeschichte erhöhten sich die Frequenzen dieser Konstruktionen und es kamen weitere Typen dazu, so zum Beispiel Frageanhängsel wie isn't it? Bei dieser Untersuchung handelt es sich um eine qualitative Studie, die auf einer ganzen Reihe von Korpora und anderen Quellen beruht, darunter zum Beispiel das Helsinki Corpus of English Texts, der Dictionary of Old English, der Middle English Dictionary und das Corpus of Historical American English. Yamaguchi (2015) verwendet ähnliche Untersuchungsmethoden, um die Entwicklung von auf Demonstrativa basierte Personenmarkierungen im Japanischen aufzuzeigen. Bei Personenmarkierungen im Japanischen handelt es sich um eine offene lexikalische Kategorie, also nicht nur um Pronomina. Im Fokus des Interesses dieser Arbeit stehen die Markierungen, die historisch auf Demonstrativa zurückgehen, wobei die genauen Prozesse dieser Entwicklung umstritten sind. Durch eine pragmatische Analyse der Sprecherinnovation argumentiert Yamaguchi dafür, dass die fraglichen Demonstrativa eine semantische Neuinterpretation erfahren haben, wodurch eine metonymische Verbindung von Raum und Person zustande kam. 
Die Arbeiten von Traugott (2016) und Yamaguchi (2015) illustrieren einen Ansatz der pragmatischen Sprachgeschichte, der sich an der theoretischen Pragmatik orientiert. Es geht um pragmatisch fundierte Erklärungsansätze für die Entwicklung von sprachlichen Elementen. Die Argumentation beruht auf einer umfangreichen Sammlung von relevanten Belegen aus verschiedenen Korpora oder Wörterbüchern, wobei der konkrete kommunikative Verwendungszusammenhang in einem sozialen Kontext nur am Rande Beachtung findet. Die folgenden Arbeiten dagegen fokussieren weniger auf die diachronen Sprachwandelprozesse, sondern auf die sozialen Kontexte der Sprachverwendung. Sie sind daher eher der soziopragmatisch ausgerichteten historischen Sprachpragmatik zuzurechnen.

Dogaru (2016), zum Beispiel, untersucht Kommunikationsverben in südsiebenbürgischen Gerichtsprotokollen des 17. Jahrhunderts. Damit nimmt sie eine enge zeitliche und textsortenspezifische Fokussierung vor und arbeitet mit einem relativ kleinen Korpus, das gut hundert Seiten umfasst. Die Kommunikationsverben teilen sich auf in Sprechaktverben wie Direktive (auffordern, befehlen), Repräsentative (behaupten) oder Expressive (gratulieren, verleumden) sowie Verba dicendi wie sagen, reden oder äußern (Dogaru 2016: 220). In der Analyse wird dargelegt, in welchen Sequenzen des Gerichtsverfahrens und von welchen Aktanten welche Kommunikationsverben eingesetzt werden. In der Initialphase des gerichtlichen Verfahrens beispielsweise, wird die Kommunikationssituation der Klageerhebung beschrieben und dafür werden typischerweise Sprechaktverben wie klagen in der Bedeutung von ,anklagen' und das damals als Verba dicendi fungierende vorgeben in der schlichten Bedeutung von, aussagen’ verwendet. Siehe Beispiel (1).

(1) Actor [=Ankläger] Michael Merten klaget wider Inct[um] [=Angeklagter] Pastorem Eqvorum undt gibt vor als sey ihm von der Stuedt ein Pferdt gestohlen worden (3 Dörfer 1698, 6ri Dogaru 2016: 223)

Die Analyse fördert eine große Zahl von Kommunikationsverben zutage und veranschaulicht ihre Verwendungsweise in einem ganz konkreten sozialen und historischen Kontext.

Einen anderen Weg gehen Jucker und Taavitsainen (2014) in ihrer Untersuchung von Komplimenten im amerikanischen Englisch. Sie basieren ihre Analyse auf dem Corpus of Historical American English (COHA) und dem Corpus of Contemporary American English (COCA). COHA umfasst Texte von 1810 bis 2009 mit einem Total von 400 Millionen 
Wörter und COCA Texte von 1990 bis 2010 und einem Total von 425 Millionen Wörter. Komplimente lassen sich allerdings nicht direkt suchen, da sie in einer unbegrenzten Vielzahl von sprachlichen Ausprägungen auftreten können. Jucker und Taavitsainen entwickeln daher die Methode der Analyse metakommunikativer Ausdrücke. In diesem speziellen Fall wurden 1741 Belegstellen des Ausdrucks compliment manuell analysiert und kategorisiert, um so Erkenntnisse über Komplimente zu erhalten, die zum expliziten Thema des Gesprächs werden, wie zum Beispiel in (2).

(2) M. de Sauvigny, it seems, had regularly sent to her all the academic eulogies of d'Alembert. One day he presented her with one to which the name of the author was not attached, but with which she was so much gratified, that she despatched a note to d'Alembert, expressing her satisfaction in animated terms, and informing him, that she considered it by far the best of all his discourses. Unluckily the eulogy was the production of Condorcet; and d'Alembert, as may be supposed, was rather offended than otherwise by her compliment. (COHA, magazine, 1831, Jucker und Taavitsainen 2014: 268)

Auf der Basis solcher Belege präsentieren Jucker und Taavitsainen statistische Trends über die Entwicklung von Komplimenten, so zum Beispiel über die prozentualen Häufigkeiten mit denen Männer oder Frauen Komplimente machen oder erhalten oder wie häufig solche Komplimente akzeptiert oder zurückgewiesen werden, auch wenn diese Zahlen sich nicht auf die effektiv gemachten, sondern nur auf die in den Daten explizit beschriebenen Komplimente beziehen.

Die Höflichkeitsforschung spielt in der aktuellen historischen Pragmatik eine bedeutende Rolle. Das Journal of Politeness Research, das sich als wesentliches Medium der internationalen Höflichkeitsforschung etabliert hat, widmete kürzlich ein Themenheft der historischen Höflichkeitsforschung in antiken Sprachen. Zwei Arbeiten seien hier speziell erwähnt. Ridealgh (2016) untersucht Texte, die mehr als 3000 Jahre alt sind. Es handelt sich um Briefe aus dem Alten Ägypten, die zwischen 1100 und 1050 vor unserer Zeitrechnung entstanden sind, die sogenannten Late Ramesside Letters. Dabei stellt sich als erstes die Frage, wie es für die moderne Forschung überhaupt möglich ist, Kategorien wie Höflichkeit in fremden und historisch fernen Kulturen zu erkennen. Lässt sich das für heutige Sprachen entwickelte Instrumentarium, insbesondere jenes von Brown und Levinson 
(1987) auf solche Texte anwenden? Oder ist es möglich, aus Darstellungen von normativem Verhalten in den Texten Rückschlüsse darüber zu ziehen, was in dieser Kultur als angemessen oder eben „höflich“ galt? Damit leistet diese Untersuchung einen Beitrag zur aktuellen Theoriedebatte in der heutigen Höflichkeitsforschung über den Nutzen von theoretischen Höflichkeitskonzepten (oft als Höflichkeit 2 bezeichnet) gegenüber dem von einer Sprachgemeinschaft selbst ausgehandelten Verständnis von Höflichkeit und angemessenem Verhalten (Höflichkeit 1 ). Die altägyptische Sprache (im Falle der Late Ramesside Letters das sog. Neuägyptische) kennt keine direkten Entsprechungen für das Wort „Höflichkeit“" Was diese Texte aber zeigen, ist ein genaues Bewusstsein für Statusunterschiede, Hierarchie und familiäre Verbindungen.

Auch Catrambone (2016) befasst sich mit einer antiken Kultur. Er untersucht Höflichkeit in den Dramen Sophokles, die im fünften Jahrhundert vor unserer Zeitrechnung entstanden sind, wobei er sich auf Interaktionen mit weiblichen Figuren in diesen Dramen fokussiert. An vielen Beispielen zeigt er, wie die Figuren einzelne der Griceschen Kooperationsmaximen verletzen, um Gesichtsbedrohungen abzuwenden. Sie verwenden indirekte Formulierungen und Andeutungen in Abhängigkeit der von Brown und Levinson postulierten Variablen der Machtverhältnisse, der Distanz zwischen den Beteiligten und der Schwere der Gesichtsbedrohung. Insbesondere die weiblichen Figuren verwenden indirekte Strategien in Interaktionen mit mächtigeren männlichen Figuren. Männliche Figuren verwenden nur dann indirekte Strategien gegenüber weiblichen, wenn sie ihnen fremd (große Distanz) oder von untergeordnetem Status (Dienstboten) sind. Weibliche Figuren unter sich verwenden nur dann indirekte Strategien, wenn es sich um eine Gesichtsbedrohung größeren Ausmaßes handelt.

Jucker (2015) verwendet ebenfalls eine literarische Quelle für seine Studie zur Höflichkeit in der englischen Renaissance. Dabei handelt es sich um Ben Jonsons Komödie Bartholomew Fair, die 1614 in London zum ersten Mal aufgeführt wurde. Die turbulente Handlung des Stücks präsentiert Figuren verschiedener sozialer Schichten, die am Jahrmarkt zum Bartholomäustag in London aufeinanderprallen. Die intrigante Welt der adligen Bewohner Londons trifft auf die zwielichtige Welt der Marktfahrer. Jucker verwendet für die Untersuchung einen diskursiven Ansatz, das heißt er verzichtet bewusst auf einen theoretischen und vorgegebenen Höflichkeitsbegriff (Höflichkeit ${ }_{2}$ ), sondern analysiert Passagen, in denen die Figuren des Stücks sich über Höflichkeit und angemessenes Verhalten 
austauschen oder in denen ihre Einschätzung von Höflichkeit oder Unhöflichkeit durch ihre Reaktion auf entsprechendes Verhalten erschlossen werden kann (Höflichkeit ${ }_{1}$ ). Während in anderen Stücken Jonsons, zum Beispiel Volpone, hinterhältige Formen der Höflichkeit verwendet werden, um unlautere und betrügerische Absichten zu überdecken (siehe Jucker 2012b), so schrecken die Figuren in Bartholomew Fair auch vor kruden Formen der Unhöflichkeit nicht zurück.

Die letzten beiden aktuellen Arbeiten, die hier kurz vorgestellt werden sollen, befassen sich aus unterschiedlicher theoretischer Perspektive mit speziellen historischen Genres. Kuna (2016) untersucht ungarische Medizinalrezepte des 16. und 17. Jahrhunderts und Schrott (2014) altspanische Beratungsdialoge. Kuna verwendet für ihre Untersuchung eine funktional-kognitive Perspektive, in der Genres als linguistische Manifestationen von sozial und kulturell determinierten kognitiven Mustern aufgefasst werden. Sie sind kontextgebundene und konventionalisierte Schemata für spezifische kommunikative Aufgaben, die im Zusammenspiel mit ähnlichen Genres untersucht werden müssen. Die untersuchten Rezepte bestehen typischerweise aus einer überschriftartigen Eröffnung, den Anweisungen mit den notwendigen Zutaten und den Details der Zubereitung und einer Wirksamkeitsbeteuerung wie im Beispiel (3).

Az kinek az szíve fáj. Vedd vizét a rozskenyérnek, és azt igyad, meggyógyul. (MOR 69, Kuna 2016: 205)

,Wem das Herz schmerzt [Eröffnung]. Nimm das Wasser von Roggenbrot und trink das [Anweisung] und werde geheilt [Wirksamkeitsbeteuerung].'

Schrotts (2014) Untersuchung der Entwicklung von Beratungsdialogen in altspanischen Texten fokussiert vor allem auf die Interaktion zwischen dem Ratsuchenden und dem Berater. Die Analyse der zwei Texte, Cantar de mio Cid (ca 1150) und Libro del Caballero Zifar (ca. 1300), kombiniert quantitative, korpuslinguistische Methoden mit einer genauen philologischen Lektüre und nutzt ein Visualisierungsprogramm, dass die lexikalische Konstellation in den Beratungsdialogen aufzeigen kann. Statistische Verfahren werden eingesetzt, um das Ausmaß der Affinität der Einheiten in lexikalischen Bündeln zu eruieren und damit linguistische Profile der Beratungsdialoge $\mathrm{zu}$ erstellen, die dann wiederum für weitere Korpusabfragen verwendet werden können. 
Diese wenigen, ausgewählten Beispiele der aktuellen Forschung in der historischen Pragmatik mögen genügen, um die große Vielfalt der Fragestellungen, Methoden, theoretischen Zugänge und der untersuchten Sprachen zu verdeutlichen. Es verwundert nicht, dass die Theoriediskussion nicht überall gleich weit fortgeschritten ist. Während einige Ansätze die innovativen Möglichkeiten von Korpusanalyse, statistischen Verfahren und Visualisierungen ausloten, verlassen sich andere Ansätze nach wie vor, und oft nicht weniger gewinnbringend, auf eher traditionelle und altbewährte Analysemethoden.

\section{Ausblick}

Als eigenständige Subdisziplin der Pragmatik konstituierte sich die historische Pragmatik in den Neunzigerjahren des letzten Jahrhunderts. Einige Philologien, so zum Beispiel die Germanistik, die Romanistik aber auch die Anglistik, verfügen über eine lange Tradition vereinzelter Arbeiten, die man aus heutiger Sicht der historischen Pragmatik zurechnen kann. Andere Philologien, zumal die Altphilologien haben die historische Pragmatik erst in den letzten Jahren für sich entdeckt. Seit 2000 gibt es eine dedizierte wissenschaftliche Zeitschrift, Journal of Historical Pragmatics, die sich ausschließlich diesem Teilgebiet der Pragmatik widmet. 2010 erschien ein Handbuch (Jucker und Taavitsainen 2010), das den damaligen Forschungsstand der historischen Pragmatik in einer umfangreichen Übersicht darstellte und 2013 ein Studienbuch (Jucker und Taavitsainen 2013) für die Hand

der Studierenden mit einer Einführung in die historische Pragmatik. Eine Übersicht über die Entwicklung der historischen Pragmatik findet sich in Taavitsainen und Jucker (2015). Das Gebiet hat sich aber inzwischen nicht nur etabliert und konsolidiert, es entwickelt sich auch rasant weiter, nicht zuletzt durch die neu hinzugekommenen Philologien, die der historischen Pragmatik ein weites Feld an Daten eröffnet, das bisher noch kaum aus pragmatischer Perspektive untersucht wurde. Mit zunehmender Weiterentwicklung der historischen Pragmatik wird sich auch die Theoriediskussion intensivieren und über die Grenzen der Einzelphilologien hinweg konsolidieren.

\section{Literatur}

Betsch, Michael (1989): Zur Entwicklung des Systems der Anredepronomina im Tschechischen 1700-1850. In: Markus Giger und Björn Wiemer (Hg.): Beiträge der Europäischen Slavistischen Linguistik (Polyslav). Bd. 1. München, 37-44. 
Breuer, Horst (1983): Titel und Anreden bei Shakespeare und in der Shakespearezeit. In: Anglia 101, 49-77.

Brown, Roger/Albert Gilman (1960): The pronouns of power and solidarity. In: Thomas A. Sebeok (Hg.): Style in Language. Cambridge, Mass., 253-276.

Brown, Roger/Albert Gilman (1989): Politeness theory and Shakespeare's four major tragedies. In: Language in Society 18.2, 159-212.

Brown, Penelope/Stephen C. Levinson (1987): Politeness. Some Universals in Language Usage. Cambridge.

Catrambone, Marco (2016): Off-record politeness in Sophocles. The patterned dialogues of female characters. In: Journal of Politeness Research 12.2, 173-195.

Chapman, Siobhan (2011): Pragmatics. Houndmills, Basingstoke.

Culpeper, Jonathan/Merja Kytö (2000): Data in historical pragmatics. Spoken discourse (re)cast as writing. In: Journal of Historical Pragmatics 1.2, 175-199.

Dogaru, Dana Janetta (2016): Kommunikationsverben in südsiebenbürgischen Gerichtsprotokollen des 17. Jahrhunderts. In: Peter Ernst/Martina Werner (Hg.): Linguistische Pragmatik in historischen Bezügen. Berlin, 219-234.

Ehrismann, Gustav (1901-1904): Duzen und Ihrzen im Mittelalter. In: Zeitschrift für deutsche Wortforschung 1, 1901, 117-149; 2, 1902, 118-159; 4, 1903, 210-248; 5, 1904, 127-220.

Finkenstaedt, Thomas (1963): 'You' and 'thou'. Studien zur Anrede im Englischen. Berlin.

Friedrich, Paul (1986): Social context and semantic feature. The Russian pronominal usage. In: John J. Gumperz/Dell Hymes (Hg.): Directions in Sociolinguistics. The Ethnography of Communication. Oxford, 270-300.

Fritz, Gerd/Manfred Muckenhaupt (1981): Du, er, sie, ihr - Anredeformen und ihre Geschichte. In: Gerd Fritz/Manfred Muckenhaupt: Kommunikation und Grammtik. Texte - Aufgaben - Analysen. Tübingen, 189-195.

Garrioch, David (1987): Verbal insults in eighteenth-century Paris. In: Peter Burke/Roy Porter (Hg.): The Social History of Language. Cambridge, 104-119.

Held, Gudrun (2005): Der Einfluss von Höflichkeit auf die mittelalterliche Briefkunst - exemplarische Überlegungen zur Entwicklung von Textstruktur und Syntax vom dictamen zur freien Briefpraxis. In: Angela Schrott/Harald Völker (Hg.): Historische Pragmatik und historische Varietätenlinguistik in den romanischen Sprachen. Göttingen, 45-61.

Jucker, Andreas H. (2012a): Pragmatics in the history of linguistic thought. In: Keith Allan/Kasia Jaszczolt (Hg.): The Cambridge Handbook of Pragmatics. Cambridge, 495-512.

Jucker, Andreas H. (2012b): "These imputations are too common, sir”. Politeness in Early Modern English dialogues. The case of Ben Jonson's Volpone, or The Fox. In: Gabriella Mazzon/Luisanna Fodde (Hg.): Historical Perspectives on Forms of English Dialogue. Mailand, 40-58.

Jucker, Andreas H. (2015): Höflichkeit im Theater der englischen Renaissance. Ben Jonson's Bartholomew Fair. In: Wolfenbütteler Renaissance-Mitteilungen 36.2, 77-88.

Jucker, Andreas H./Irma Taavitsainen (Hg.) (2010): Historical Pragmatics. Berlin/New York. Jucker, Andreas H./Irma Taavitsainen (2013): English Historical Pragmatics. Edinburgh. 
Jucker, Andreas H./Irma Taavitsainen (2014): Complimenting in the history of American English. A metacommunicative expression analysis. In: Irma Taavitsainen/Andreas H. Jucker/Jukka Tuominen (Hg.): Diachronic Corpus Pragmatics. Amsterdam, 257-276.

Kryk-Kastovsky, Barbara (2000): Representations of orality in Early Modern English trial records. In: Journal of Historical Pragmatics 1.2, 201-230.

Kuna, Ágnes (2016): Genre in a functional cognitive framework. Medical recipe as a genre in 16th and 17th century Hungarian In: Ninke Stukker/Eilnrtz Spooren/Gerard Steen (Hg.): Genre in Language, Discourse and Cognition. Berlin/New York, 193-224.

Lebsanft, Franz (1987): Le problème de mélange du 'tu' et du 'vous' en ancien français. In: Romania 108, $1-19$.

Lebsanft, Franz (1988): Studien zu einer Linguistik des Grußes. Sprache und Funktion der altfranzösischen Grußformeln. Tübingen.

Lötscher, Andreas (1981): Zur Sprachgeschichte des Fluchens und Beschimpfens im Schweizerdeutschen. In: Zeitschrift für Dialektologie und Linguistik 48, 145-160.

Metcalf, George J. (1938): Forms of Address in German (1500-1800). St. Louis.

Montagu, Ashley (1967): The Anatomy of Swearing. Philadelphia.

Nevalainen, Terttu/Helena Raumolin-Brunberg (1995): Constraints on politeness. The pragmatics of address formulae in Early English correspondence. In: Andreas H. Jucker (Hg.): Historical Pragmatics. Pragmatic Developments in the History of English. Amsterdam/Philadelphia, 541-601.

Ridealgh, Kim (2016): Polite like an Egyptian? Case studies of politeness in the Late Ramesside Letters. In: Journal of Politeness Research 12.2, 245-266.

Salmon, Vivian (1967): Elizabethan colloquial English in the Falstaff plays. Leeds Studies in English. New Series 1, 37-70. Reprinted in: Vivian Salmon/Edwina Burness (Comp.) (1987): A Reader in the Language of Shakespearean Drama. Amsterdam/Philadelphia.

Schlieben-Lange, Brigitte (1976): Für eine historische Analyse von Sprechakten. In: H. Weber/Harald Weydt (Hg.): Sprachtheorie und Pragmatik. Tübingen, 113-119.

Schlieben-Lange, Brigitte/Harald Weydt (1979): Streitgespräch zur Historizität von Sprechakten. In: Linguistische Berichte 60, 65-78.

Schwitalla, Johannes (2000): Wandlungen eines Mediums. Sprachliche Merkmale öffentlicher Briefe von Laien in der Reformationszeit. In: Irmhild Barz/Ulla Fix/Marianne Schröder/Georg Schuppner (Hg.): Sprachgeschichte als Textsortengeschichte. Festschrift zum 65. Geburtstag von Gotthard Lerchner. Frankfurt am Main, 261-279.

Schrott, Angela (2014): A matter of tradition and good advice: Dialogue analysis and corpus pragmatics in Old Spanish texts. In: Irma Taavitsainen/Andreas H. Jucker/Jukka Tuominen (Hg.): Diachronic Corpus Pragmatics. Amsterdam, 303-327.

Simon, Horst (2003): From pragmatics to grammar: Tracing the development of respect in the history of the German pronouns of address. In: Irma Taavitsainen/Andreas H. Jucker (Hg.): Diachronic Perspectives on Address Term Systems. Amsterdam/Philadelphia, 85-123.

Stroebe, Klara (1911): Altgermanische Grussformen. Heidelberg. 
Taavitsainen, Irma/Andreas H. Jucker (2015): Twenty years of historical pragmatics. Origins, developments and changing thought styles. In: Journal of Historical Pragmatics 16.1, 1-25.

Taavitsainen, Irma/Andreas H. Jucker/Jukka Tuominen (Hg.) (2014): Diachronic Corpus Pragmatics. Amsterdam.

Traugott, Elisabeth Closs (2016): On the rise of types of clause-final pragmatic markers in English. In: Journal of Historical Pragmatics 17.1, 26-54.

Yamaguchi, Toshiko (2015): The rise of demonstrative-based first/second-person markers in the history of Japanese. A speaker subjectivity account. In: Journal of Historical Pragmatics 16.2, 250-276.

Andreas H. Jucker, Universität Zürich 